\title{
Next Generation 4G WiMAX Networks - IEEE 802.16 Standard:
}

\author{
Shantanu Pathak ${ }^{1}$ and Shagun Batra ${ }^{2}$ \\ Department of Telecommunication, SRM University, Chennai, India \\ ${ }^{1}$ shantanupathak614@gmail.com, ${ }^{2}$ batra.shagun@gmail.com
}

\begin{abstract}
This paper gives an overview about the various PHY and MAC layer specification which define the IEEE 802.16 standard and which define the Fixed WiMAX (Ver. 802.16d-2004) and Mobile WiMAX (Ver. 802.16e-2005) network scenarios. We also discuss about the various updates in the Advanced Air Interface update $(802.16 m$ - 2011), which aims at fulfilling the $4 G$ requirements as put forward by the ITU IMT-Advanced requirements.
\end{abstract}

\section{KEYWORDS}

WiMAX, IEEE, 802.16m, 802.16e, OFDMA, MIMO, AMC, PHY, MAC, QoS,

\section{INTRODUCTION}

$4 \mathrm{G}$ is the term used to refer to the next wave of high-speed mobile technologies that will be used to replace current $3 \mathrm{G}$ networks. As per the standards of $4 \mathrm{G}$ under ITU (International Telecommunication Union), the new technology must provide peak speed for Internet communication at $100 \mathrm{Mbps}$ for high mobility users and $1 \mathrm{Gbps}$ for low mobility users. $4 \mathrm{G}$ also promises faster cellular system. The fourth-generation will inter-operate with third generation systems; it also intends to integrate fixed wireless access (FWA), wireless local area network (WLAN), wireless local loop (WLL) and personal area network (PAN), to provide fully IP-based wireless internet. The two top contenders are LTE and WiMAX, both of which are IP based networks that are built from similar, yet incompatible, technologies.

WiMAX is a wireless digital communications system, also known as IEEE 802.16 that is intended for wireless "metropolitan area networks". WiMAX can provide broadband wireless access (BWA) up to 30 miles $(50 \mathrm{~km})$ for fixed stations, and 3 - 10 miles $(5-15 \mathrm{~km})$ for mobile stations. It is maintained by the WiMAX Forum industry alliance. The Forum promotes and certifies compatibility and interoperability of products based on the IEEE 802.16 standards.

The IEEE 802.16 standard was developed in its first version, as a means of fixed wireless broadband access in the frequency range 10-66 GHZ. The standard has undergone a number of updates such as, 802.16a, 802.16c, and also the fixed WiMAX standard, 802.16d (802.16-2004), the mobile WiMAX 802.16e standard (802.16-2005) which each specifies air interface for fixed and mobile WiMAX standards along with related functions. 802.16-2009 provides a rollup of all updates till 2009 under a single update and provides an air interface for fixed and mobile broadband wireless access.

Sundarapandian et al. (Eds): CoNeCo,WiMo, NLP, CRYPSIS, ICAIT, ICDIP, ITCSE, CS \& IT 07, pp. 507-518, 2012. (C) CS \& IT-CSCP 2012 
The standard specifies the air interface of BWA, which include the Medium Access Control (MAC) and Physical (PHY) layers. The chief advancement of the PHY layer includes orthogonal frequency-division multiplexing (OFDM), in which multiple access is achieved by assigning a subset of subcarriers for each individual user. With advances in standards including the 802.16e support for advanced antenna system (AAS) through MIMO technology, which uses multiple antennas at both transmitter and receiver, which sets up a multiple data stream on the same channel, increasing the data capacity of the channel to a great extent. The MAC layer uses a scheduling algorithm which determines access between subscriber station and base station. It also provides control of Quality of Service (QoS) parameters through the base station.

The next evolutionary update of the IEEE 802.16 standard is the IEEE $802.16 \mathrm{~m}(802.16-2011)$ also known as WiMAX rel 2.0.Building upon the IEEE 802.16e or mobile WiMAX, this standard is accepted candidate for the IMT-Advanced specifications provided by the ITU. Like its previous version, IEEE 802.16m uses OFDMA as multiple access scheme, with support for TDD, FDD and half duplex FDD. The fundamental concept of WiMAX remains the same, but in order to meet the IMT-Advanced specifications many technical updates are provided, which aim for higher efficiencies in PHY and MAC layer and environment optimised deployments, as well as high data rates and low packet latencies.

\section{WIMAX IEEE 802.16 PHYSICAL LAYER:}

The IEEE 802.16 physical layer uses a number of legacy technologies which include Orthogonal Frequency Division Multiplexing (OFDM), Time Division Duplexing (TDD), Frequency Division Duplexing (FDD), Adaptive Modulation and Coding (AMC), and Adaptive Antenna System (AAS). AMC with 16 different modulation and coding schemes is used to manage fine granularity of link adaption mechanism. Multi-Carrier operation is used to enable - virtuall carriers up to $100 \mathrm{MHz}$ wide and for deployments in radio spectrum for carriers other than 5,10 and $20 \mathrm{MHZ}$.

\subsection{OFDM-based air interface:}

The WiMAX air interface (PHY Layer) is based on Orthogonal Frequency Division Multiplexing. To maintain orthogonality between the individual carriers the symbol period must be reciprocal of the carrier spacing. OFDM is a form of multi-carrier modulation, which divides a high bit stream into a number of low bit streams, which are each modulated by separate carriers called subcarriers or tones. These modulation schemes generally remove Inter Symbol Interference by keeping the symbol duration large. But for high data rate systems where the symbol duration is small, the high bit stream is splitted into several parallel streams increasing the symbol duration of each stream. The sub-carriers are chosen such that they are orthogonal; hence there is no mutual interference among the signal streams.

The IEEE 802.16 standard supports number of physical layer specifications. The first version of this standard only supported single carrier modulation in in licensed spectrum of 10-66 GHZ in LOS operations. With addition of OFDM, OFDMA and scalable OFDMA, IEEE 802.16 operation also includes NLOS and also provides mobility.

The range of frequencies supported in the licensed as well as unlicensed bands for IEEE 802.16 is as described below:

- $\quad 10-66 \mathrm{GHz}$ licensed band - IEEE 802.16-2004 defines the PHY layer for the 10-66 GHz licensed spectrum. Operation in LOS environment.

- 2-11 GHz licensed and licensed exempt - In this frequency bands, both licensed and licensed exempt bands are addressed. Additional features help in operation of NLOS and LOS environment also reduce the effects of multi path propagation. 
- WirelessMAN-SC PHY mode of IEEE 802.16-2004 supports operation in the 10-66 GHz frequency band. At these frequencies LOS operation is more practical. This mode is an adaptive modulation scheme (QPSK, 16-QAM, 64-QAM) on a single carrier. Duplexing support is by TDD or FDD.

- The WirelessMAN-OFDM PHY is based on Orthogonal Frequency Division Multiplexing (OFDM) modulation. It has been designed for NLOS operation in frequency band 2-11 GHZ. It uses 256-point transform in OFDM access is by TDMA. Operates on license exempt bands

\begin{tabular}{|c|c|c|c|}
\hline Designation & Band of Operation & $\begin{array}{l}\text { Duplexing } \\
\text { Mode }\end{array}$ & Notes \\
\hline WirelessMAN-SC & $10-66 \mathrm{GHz}$ & TDD, FDD & Single Carrier \\
\hline WirelessMANSCa & $\begin{array}{l}\text { 2-11 GHz Licensed } \\
\text { band }\end{array}$ & TDD, FDD & Single Carrier technique for NLOS \\
\hline WirelessMAN & $2-11 \mathrm{GHz}$ & TDD & OFDM for NLOS operation \\
\hline OFDM & Licensed band & FDD & \\
\hline WirelessMAN & $2-11 \mathrm{GHz}$ & TDD & OFDM Broken into subgroups to \\
\hline OFDMA & Licensed band & FDD & $\begin{array}{l}\text { provide multiple access in a single } \\
\text { frequency band }\end{array}$ \\
\hline WirelessHUMAN & $\begin{array}{c}\text { 2-11 GHz Licensed } \\
\text { Band Exempt }\end{array}$ & TDD & $\begin{array}{l}\text { May be SC, OFDM, OFDMA. Must } \\
\text { include Dynamic Frequency } \\
\text { Selection to mitigate interference }\end{array}$ \\
\hline
\end{tabular}

IEEE 802.16 air interface specifications

WirelessMAN-OFDMA is among the three specifications defined in IEEE 802.16 family for applications below $11 \mathrm{GHz}$. The IEEE 802.16e-2005 amendment was developed to extend the 802.16 Air Interface Standard to cover mobile applications. This uses orthogonal frequencydivision multiple access with a 2048-point transform and is designed for NLOS operation in the frequency bands below $11 \mathrm{GHz}$. In the OFDMA mode, the active subcarriers are divided into subsets of subcarriers, where each subset is termed a sub channel. In the downlink, a sub channel may be intended for different receiver(s); In the uplink a sub channel may be assigned for different transmitter(s).This sub channels may not be adjacent.

The 802.16e-2005 standard gives three sub channel allocation based on usage environments:

- Subcarriers can be scattered throughout the frequency channel range. This is referred to as fully used sub channelization or FUSC.

- Several scattered clusters of subcarriers can be used to form a sub channel. This is referred to as partially used sub channelization or PUSC.

- Sub channels can be composed of contiguous groups of subcarriers. This is referred to as adaptive modulation and coding or AMC. 


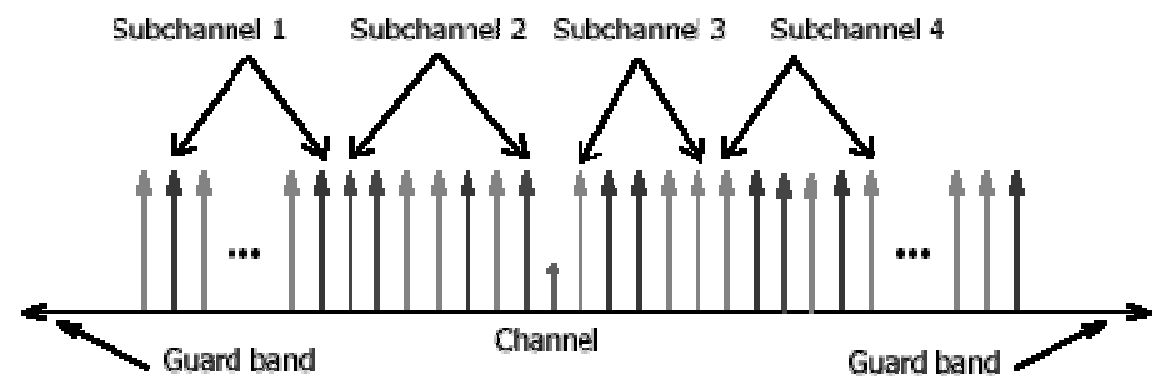

OFDMA sub channel description

\subsubsection{Support for heterogeneous users in IEEE 802.16m update:}

One of the main objectives of future wireless system is to able to provide efficient and flexible services to users of different needs. For some users low data rates but a reliable link is preferred, while some users require high data rates, even if delays from retransmissions can occur. Moreover the co-existence should have low overheard on the systems. Hence under the IEEE $802.16 \mathrm{~m}$ update, frame structure has been modified with reference to the 802.16e. The frame structure of the 80216e consists of a downlink and an uplink part separated by OFDMA symbols of variable size. The frame structure under $802.16 \mathrm{~m}$ is given below:

Data is organized into a hierarchy of superframes, frames, subframes and OFDM symbols. Each superframe last $20 \mathrm{~ms}$ and contains four $5 \mathrm{~ms}$ frames. Each of these $5 \mathrm{~ms}$ frames contains 8 subframes. However if the channel size is $7 \mathrm{MHZ}$ in which case frames contain 6 subframes, or 8.75 MHZ, in which case frames contain 7 subframes. Subframes fall into one of four categories, three of which are employed as part of $802.16 \mathrm{~m}$ and a fourth that is included for legacy operation with 802.16-2009 devices operating on $8.75 \mathrm{MHz}$ channels. There are four types of subframes: Type- 1 has 6 OFDMA symbols; Type-2 has 7 OFDMA symbols; Type-3 has 6 OFDMA symbols; Type -4 has 9 OFDMA symbols and is included for legacy operation with 802.16-2009 devices operating on $8.75 \mathrm{MHz}$ channels.

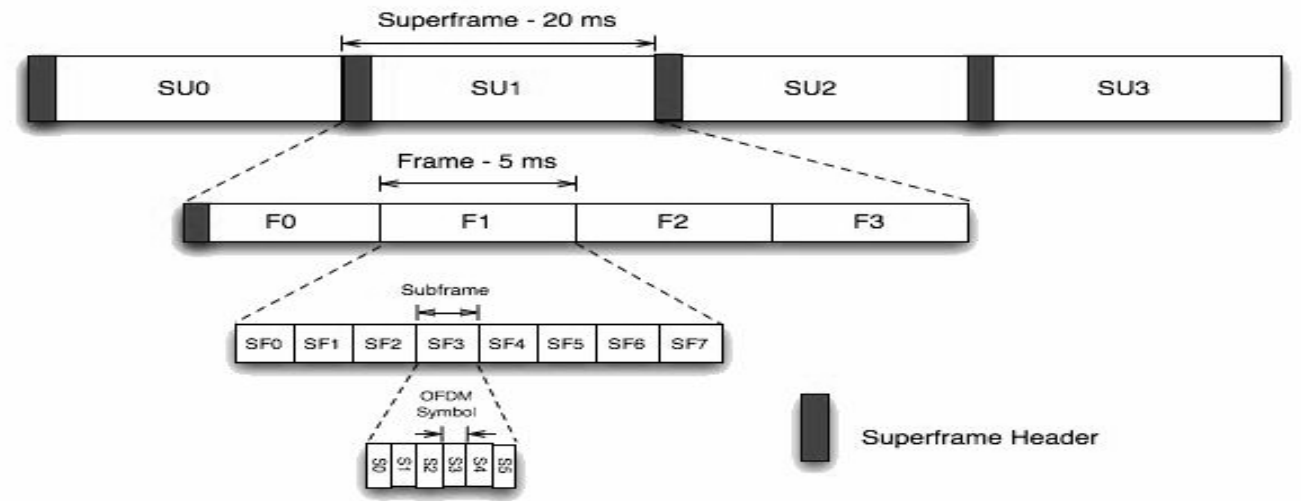

Frame structure with Type-1 subframe

\subsection{Adaptive Antenna Systems (AAS):}

Adaptive Antenna Systems (AAS) uses beam-forming techniques to focus the wireless beam between the base station and the subscriber. Under beam-forming multiple antennas and multiple signals are employed, which then shape the beam with the intent of improving transmission to the desired station. The result is reduced interference because the signal going to the desired user is increased and the signal going to other users is reduced. Dynamic Frequency Selection (DFS) 
schemes are applied in which air waves are first scanned to determine where interference doesn't occur, and specific frequencies in that area where no interference occurs is selected. These are achieved in WiMAX through the use of MIMO (multiple-input multiple output) communication schemes. MIMO uses multiple antennas at both transmitter and receiver, which sets up a multiple data stream on the same channel, increasing the data capacity of the channel to a great extent. With multiple antennas at both transmitter and receiver, the transmitter and receiver can coordinate to move to an open frequency if/when interference occurs. In application, the MIMO technology can be divided based on Open Loop MIMO techniques and Closed Loop MIMO techniques. MIMO systems that do not rely on knowledge of the channel responses at the transmitter are open loop. Closed loop is the case when the channel state information (CSI) is available at the transmitter through some form of feedback mechanism.

\subsubsection{Extended Use of MIMO under 802.16m:}

IEEE $802.16 \mathrm{~m}$ supports several advanced multi-antenna techniques including single and multiuser MIMO (spatial multiplexing and beam-forming) as well as a number of transmit diversity schemes. In single-user MIMO (SU-MIMO) scheme only one user can be scheduled over one resource unit, while in multi-user MIMO (MU-MIMO), multiple users can be scheduled in one resource unit.Moreover, although some other MIMO features of $802.16 \mathrm{e}$, such as closed-loop MIMO, have not appeared in Mobile WiMAX profiles yet, it is expected that they will be included in new $802.16 \mathrm{~m}$-based systems. More specifically, it has been already decided to support closed-loop MIMO using Channel Quality Information, Precoding Matrix Index and rank feedback in future systems.

\begin{tabular}{|c|c|c|}
\hline Requirement & IEEE 802.16e & IEEE802.16m \\
\hline Aggregate Data Rate & $63 \mathrm{Mbps}$ & $\begin{array}{l}100 \text { Mbps for mobile } \\
\text { stations, } 1 \text { Gbps for } \\
\text { fixed }\end{array}$ \\
\hline $\begin{array}{l}\text { Operating } \\
\text { Frequency }\end{array}$ & $\begin{array}{l}2.3 \quad \mathrm{GHz}, \quad 2.5-2.7 \\
\mathrm{GHz}, 3.5 \mathrm{GHz}\end{array}$ & $<6 \mathrm{GHz}$ \\
\hline Duplexing Schemes & TDD and FDD & TDD and FDD \\
\hline MIMO support & $\begin{array}{l}\text { up to } 4 \text { streams, no } \\
\text { limit on antennas }\end{array}$ & $\begin{array}{l}4 \text { or } 8 \text { streams, no limit } \\
\text { on antennas }\end{array}$ \\
\hline Coverage & $10 \mathrm{~km}$ & $\begin{array}{l}3 \mathrm{~km}, 5-30 \mathrm{~km} \text { and } 30- \\
100 \mathrm{~km}\end{array}$ \\
\hline $\begin{array}{l}\text { Handover Inter- } \\
\text { frequency } \\
\text { Interruption Time }\end{array}$ & $35-50 \mathrm{~ms}$ & depending on scenario \\
\hline $\begin{array}{l}\text { Handover Intra- } \\
\text { frequency } \\
\text { Interruption Time }\end{array}$ & Not Specified & $30 \mathrm{~ms}$ \\
\hline $\begin{array}{l}\text { Handover between } \\
802.16 \text { standards } \\
\text { (for corresponding } \\
\text { mobile station) }\end{array}$ & $\begin{array}{l}\text { From } 802.16 \mathrm{e} \\
\text { serving BS to } \\
802.16 \mathrm{e} \text { target BS }\end{array}$ & $100 \mathrm{~ms}$ \\
\hline
\end{tabular}

IEEE $802.16 \mathrm{e}$ and $802.16 \mathrm{~m}$ Requirements

Single-user MIMO (SU-MIMO) schemes are used to improve the link performance, by providing robust transmissions with spatial diversity, or large spatial multiplexing gain and peak data rate to a single MS, or beamforming gain. Both open-loop SU-MIMO and closed-loop SU-MIMO is supported in $16 \mathrm{~m}$. For open-loop SU-MIMO, both spatial multiplexing and transmit diversity 
schemes are supported. For closed-loop SU-MIMO, codebook based precoding is supported for both TDD and FDD systems.

Multi-user MIMO (MU-MIMO) schemes are used to enable resource allocation to communicate data to two or more mobile stations. MU-MIMO enhances the system throughput. Multi-user transmission with one stream per user is supported in MU-MIMO mode. MU-MIMO includes the MIMO configuration of 2 Transmit antennas to support up to 2 users, and 4 Transmit or 8 Transmit antennas to support up to 4 users. Both unitary and non-unitary MU-MIMO linear precoding techniques are supported. For open-loop MU-MIMO, channel quality indicator (CQI) and preferred stream index feedback may be transmitted to assist the base station's scheduling, transmission mode switching, and rate adaptation. The CQI is frequency dependent. For closedloop multi -user MIMO, codebook based precoding is supported for both TDD and FDD systems. CQI and PMI feedback can be transmitted by the mobile station to assist the base station's scheduling, resource allocation, and rate adaptation decisions.

\subsection{Adaptive Modulation and Coding (AMC):}

WiMAX supports a variety of modulation and coding schemes. These schemes are adaptive, and can hence be made to vary its parameters, according to the prevalent conditions. WiMAX modulation and coding can be made to change on a burst by burst basis per link. The coding and modulation schemes maintain a steady signal strength by using different schemes over increasing distance; this is achieved by decreasing throughput over range so as to provide the best Quality of Service (QoS) possible over distance. Also, a technique called Dynamic Bandwidth Allocation (DBA) is used, which monitors the network for interference or reduction in signal strength, the base station allocates more bandwidth to the afflicted stream. To determine the required WiMAX modulation and coding scheme the channel quality feedback indicator is used. The mobile (UE) can provide the base station (BS) with feedback on the downlink channel quality and for the uplink, the base station can estimate the channel quality, based on the received signal quality.

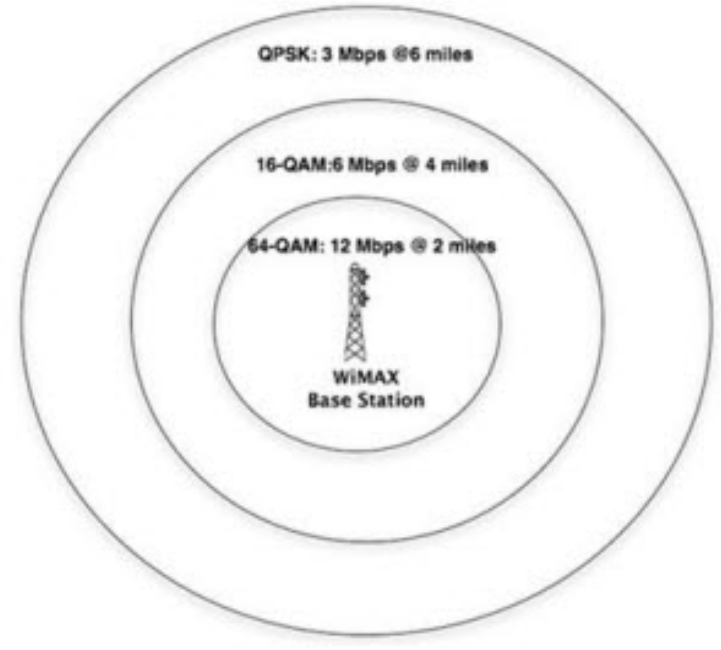

Adaptive Modulation under WiMAX

The various modulation schemes used under WiMAX:

Downlink: BPSK, QPSK, 16 QAM, 64 QAM; BPSK optional for OFDM-PHY.

Uplink: BPSK, QPSK, 16 QAM; 64 QAM optional.

The various coding schemes used under WiMAX:

Downlink: convolutional codes at 1/2, 2/3, 3/4, 5/6 
Uplink: convolutional codes at 1/2,2/3, 3/4, 5/6

In telecommunication, convolutional codes are like error-correcting codes, in which each m-bit information bit is to be transformed into an $n$-bit symbol where $m / n$ is the code rate $(n \geq m)$.the transformation is a function of last information $\mathrm{k}$ information symbol, where $\mathrm{k}$ is a the constraint length of the symbol.

\section{WIMAX IEEE 802.16 MAC LAYER:}

Medium Access Control or MAC layer lies above the PHY layer of the open system interconnect (OSI) protocol stack layers. Its primary objective is to provide an interface between the higher transport layers and the lower physical layer. The MAC layer takes packets from the upper layer, these packets are called MAC service data units (MSDUs).and organizes them into MAC protocol data units (MPDUs) for transmission over the air. For received transmissions, the MAC layer does the reverse. . The MAC protocols may use either distributed multiple access techniques or centrally coordinates the access for all devices, or even uses a combination of two.

The IEEE 802.16 MAC protocol was designed for point-to-multipoint (PMP) broadband wireless access application, and is based on collision sense multiple access with collision avoidance (CSMA/CA). But to accommodate the more demanding physical environment and different service requirements MAC supports mesh, rather than only point to multipoint network architectures. The IEEE 802.16-2004 and IEEE 802.16e-2005 MAC design includes a convergence sublayer that can interface with a variety of higher-layer protocols, such as ATM TDM Voice, Ethernet, IP, and any unknown future protocol. IEEE 802.16 standard is designed to work with outdoor networks, both in urban as well as rural areas. The MAC incorporates several features suitable for a broad range of applications at different mobility rates, such as, privacy key management (PKM) for MAC layer security; broadcast and multicast support; Manageability primitives; high-speed handover and mobility management primitives; three power management levels, normal operation, sleep and idle; Header suppression, packing and fragmentation for efficient use of spectrum. These features along with benefits scalable OFDMA make 802.16 suitable for high-speed data and bursty or isochronous IP multimedia applications.

The IEEE 802.16 MAC layer is composed of three sub-layers: Service Specific Convergence Sub-layer (CS); MAC Common Part Sub-layer (CPS); and the Security Sub-layer. Each sub-layer has a specific function to perform.

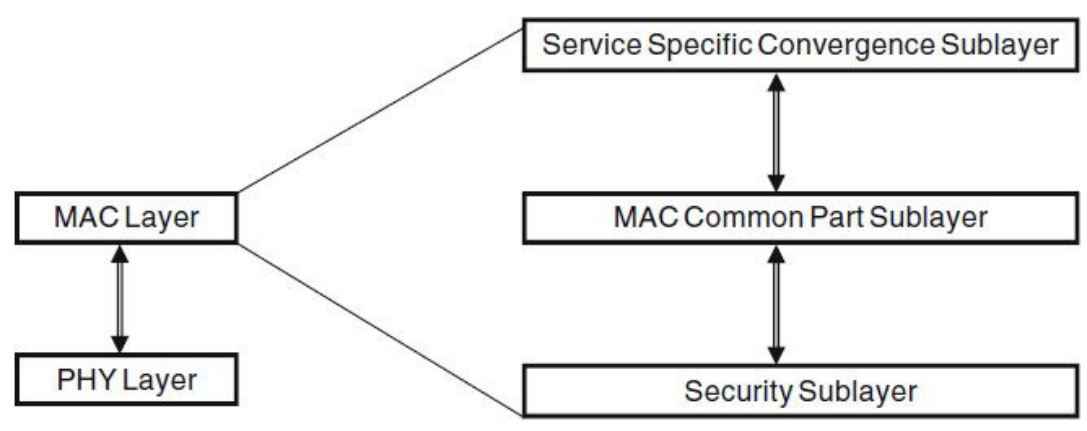

IEEE 802.16 MAC LAYER
Protocol Specific, i.e

ATM,Ethernet,

Internet Protocol

Packing

Fragmentation,

ARQ QoS

Authentication, Key

Exchange,

Encryption 
The Service-Specific Convergence Sub-layer (CS) provides any transformation or mapping of external network data, received through the CS service access point (SAP), into MAC SDUs received by the MAC Common Part Sub-layer (CPS) through the MAC SAP.

The core part of the IEEE 802.16 MAC is the MAC CPS, as it provides the core MAC functionality of system access, bandwidth allocation, connection establishment, and connection maintenance. Also functions like uplink scheduling, bandwidth request and grant, connection control, and automatic repeat request (ARQ) are also defined. Communication between the CS (Convergence Sub-layer) and the MAC CPS are maintained by MAC Service Access Point (MAC SAP).The four basic actions of communication that is creation of connection, modification of connection, deletion of connection and transport of data over the connection is done at this layer. The MAC also contains a separate security sublayer providing authentication, secure key exchange, and encryption. Data, PHY control, and statistics are transferred between the MAC CPS and the PHY via the PHY SAP (which is implementation specific).

\subsection{Quality of Service (QoS) Service classes:}

QoS or Quality of Service is a key element in the delivery of service over the IEEE 802.16 medium. IEEE 802.16 defines five QoS service classes:

Unsolicited Grant Scheme (UGS), Extended Real Time Polling Service (ertPS), Real Time Polling Service (rtPS), Non Real Time Polling Service (nrtPS), and Best Effort Service (BE). Each of these has its own QoS parameters such as minimum throughput requirement and delay/jitter constraints.

\begin{tabular}{|c|c|c|}
\hline QoS & Pros & Cons \\
\hline UGS & $\begin{array}{l}\text { No overhead. Meet guaranteed } \\
\text { latency for real- time service }\end{array}$ & $\begin{array}{l}\text { Bandwidth may not be } \\
\text { utilized fully since } \\
\text { allocations are granted } \\
\text { regardless of current need }\end{array}$ \\
\hline ertPs & $\begin{array}{l}\text { Optimal latency and data } \\
\text { overhead efficiency }\end{array}$ & $\begin{array}{l}\text { Need to use the polling } \\
\text { mechanism(to meet the } \\
\text { delay guarantee) and a } \\
\text { mechanism to let the BS } \\
\text { know when the traffic } \\
\text { starts during silent perios }\end{array}$ \\
\hline rtPS & $\begin{array}{l}\text { Optimal data transport } \\
\text { efficiency }\end{array}$ & $\begin{array}{l}\text { Require the overhead of } \\
\text { bandwidth request and the } \\
\text { polling latency(to meet the } \\
\text { delay guarantee) }\end{array}$ \\
\hline nrtPS & $\begin{array}{l}\text { Provide efficient service for } \\
\text { non-real-time traffic with } \\
\text { minimum reserved rate }\end{array}$ & N/A \\
\hline $\mathrm{BE}$ & $\begin{array}{l}\text { Provide efficient service for } \mathrm{BE} \\
\text { traffic }\end{array}$ & $\begin{array}{l}\text { No service guarantee, } \\
\text { some connections may } \\
\text { starve for long period of } \\
\text { time }\end{array}$ \\
\hline
\end{tabular}

\section{Comparison of QoS Classes}

UGS: This class is used for real time services such as VoIP or for other application where WiMAX is used for fixed lines replacement.

rtPS: This class is also used for real time services including video streaming. It is also used for enterprise access services. This class offers variable bit rate but with guaranteed minimums for data rate and delay. 
ertPS: This class is used for applications where variable packet sizes are used. E.g. Skype

nrtPS: This class is used for services where a guaranteed bit rate is required but the latency is not critical. It might be used for various forms of file transfer.

BE: This class is used for internet services such as email and browsing. Data packets are carried as space become available.

However, for non-real-time traffic, traffic priority is also one the QoS parameters that can differentiate among different connections or subscribers within the same service class.

\subsection{Scheduler:}

Scheduling is the chief feature of the MAC layer that helps assure variable QoS. The Scheduler works as distributor of allocated resources to mobile stations (MS). The allocated resource can be defined as the number of slots and then these slots are mapped into a number of subchannels (each subchannel is a group of multiple physical subcarriers) and time duration (OFDM symbols).

The mapping process from logical subchannel to multiple physical subcarriers is called a permutation. Partial Used

Subchannelization (PUSC), Fully Used Subchannelization (FUSC), Adaptive Modulation and Coding (band-AMC) are different permutation modes. The term band-AMC distinguishes the permutation from adaptive modulation and coding (AMC) MCS selection procedure. Basically there are two types of permutations: distributed and adjacent. The distributed subcarrier permutation is suitable for mobile users while adjacent permutation is for fixed (stationary) users. The Scheduler logically assigns the resources in terms of number of slots, it decides the assignment of sub carrier allocation. . In systems with Single Carrier PHY, the scheduler assigns the entire frequency channel to a MS, also deciding how to allocate the number of slots in a frame for each user.

In systems with OFDM PHY, the scheduler considers the modulation schemes for various subcarriers and decides the number of slots allocated. In systems with OFDMA PHY, the scheduler needs to take into consideration the fact that a subset of subcarriers is assigned to each user. Physically the scheduler decides which subchannels and time intervals are suitable for different users. The goal is to minimize power consumption, to minimize bit error rate and to maximize the total throughput.

\subsection{Hybrid Automatic Repeat Request (HARQ):}

HARQ or Hybrid Automatic Repeat Request is a system used by $802.16 \mathrm{~m}$ to ensure all packets are transmitted and correctly received. In this system, each frame is sent; the sender waits until it received an ACK (acknowledgement) before sending the next frame. Multiple HARQ channels can run in parallel (up to 16), mitigating the performance hit of waiting for an ACK before sending more data.

IEEE 802.16e used a different version of HARQ, which is supported in $802.16 \mathrm{~m}$. The old version is called Chain Combining, where exactly the same data is retransmitted when an ACK is not received.802.16m uses a different variation of HARQ known as Incremental Redundancy, where data is retransmitted with different encoding. 


\section{IMT-ADVANCED FEATURES UNDER 802.16M (WiMAX rel 2.0 Systems):}

\subsection{Relays:}

Intelligent relays are an effective technology to provide cost effective methods of delivering high data rate and avoid coverage holes in deployments areas. In addition, upgrading the networks in order to support higher data rates is equivalent to an increase of signal-to-interference plus noise ratio (SINR) at the receivers' front-end.

Relay stations are capable of decoding and forwarding the signals from source to destination through radio interface. Relay stations do not need a wire backhaul. The system performance can be further improved by intelligent resource scheduling and cooperative transmissions in systems employing intelligent relays.

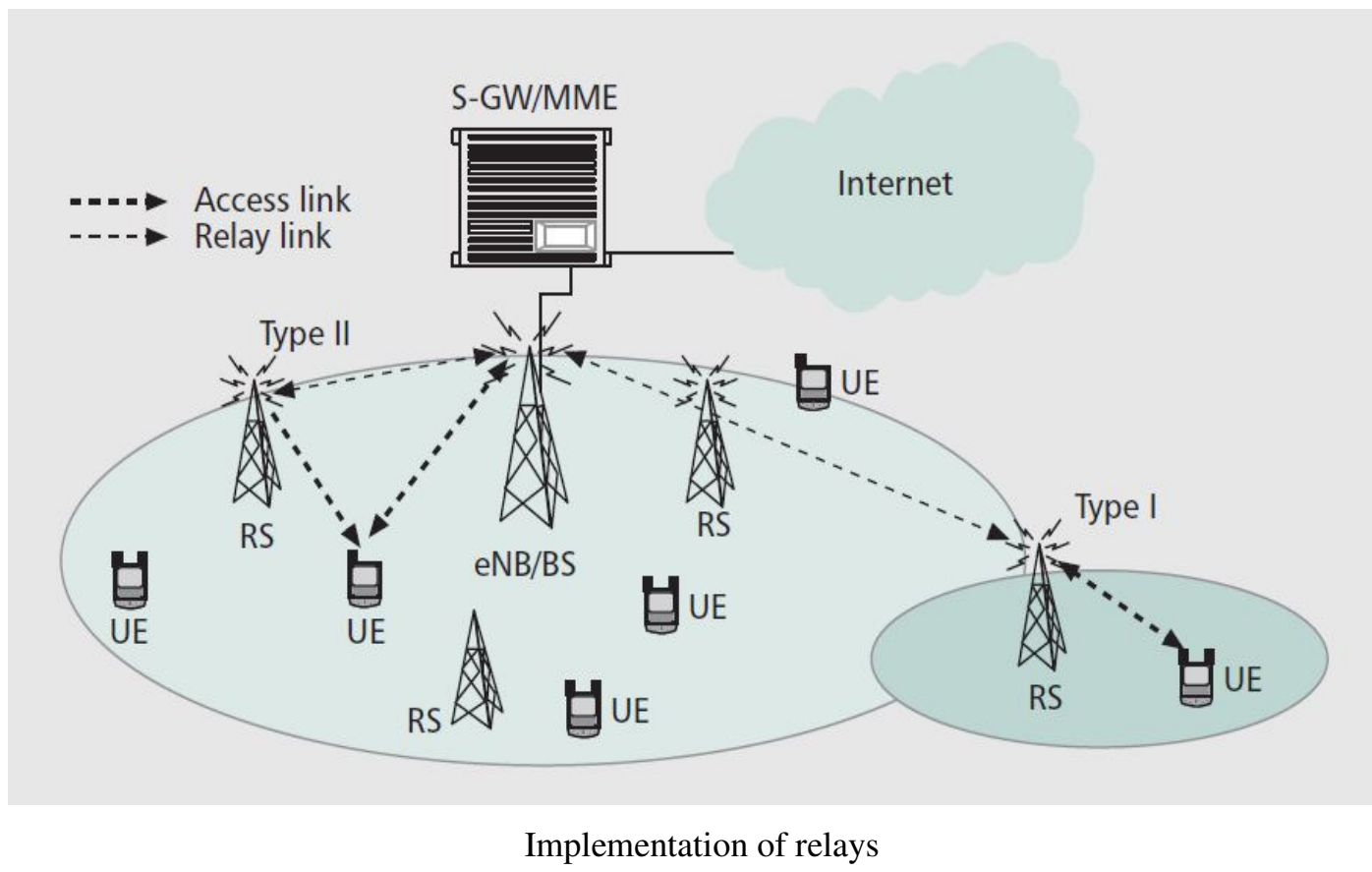

Two different relay modes are defined in this standard, transparent mode and Non- transparent mode.

The transparent relay mode increases the throughput which facilities capacity increases within the Base Station (BS) coverage area. It has no support to coverage extension because it does not forward framing information to BS. It is operated in two hop network topology and supports centralized scheduling only as scheduling is done only in BS.

The Non transparent relay mode is to increase the coverage extension of BS, here Relay Stations (RS) generate its own framing information and forward it to Mobile Station (MS). It operates is 2 or more hops and uses centralized or distributed scheduling mode, as scheduling is done in BS and RSs.

\subsection{Femtocell and Self Organizing Networks:}

Femtocells in $802.16 \mathrm{~m}$ are low powered access points, to provide access to closed or open group of users as configures by the subscribers. Femtocells are normally connected to service provider's 
network. For femtocells base stations which can support relay links, it may establish air interface connection with overlapping macrocell base station for exchange of control message.

Femtocell BS is intended to serve public users, like public WiFi hot spot, or to serve closed subscriber group (CSG) that is a set of subscribers authorized by the femtocell BS owner or the service provider. CSG can be modified by the service level agreement between the subscriber and the access provider.

\section{How Femtocells Work}

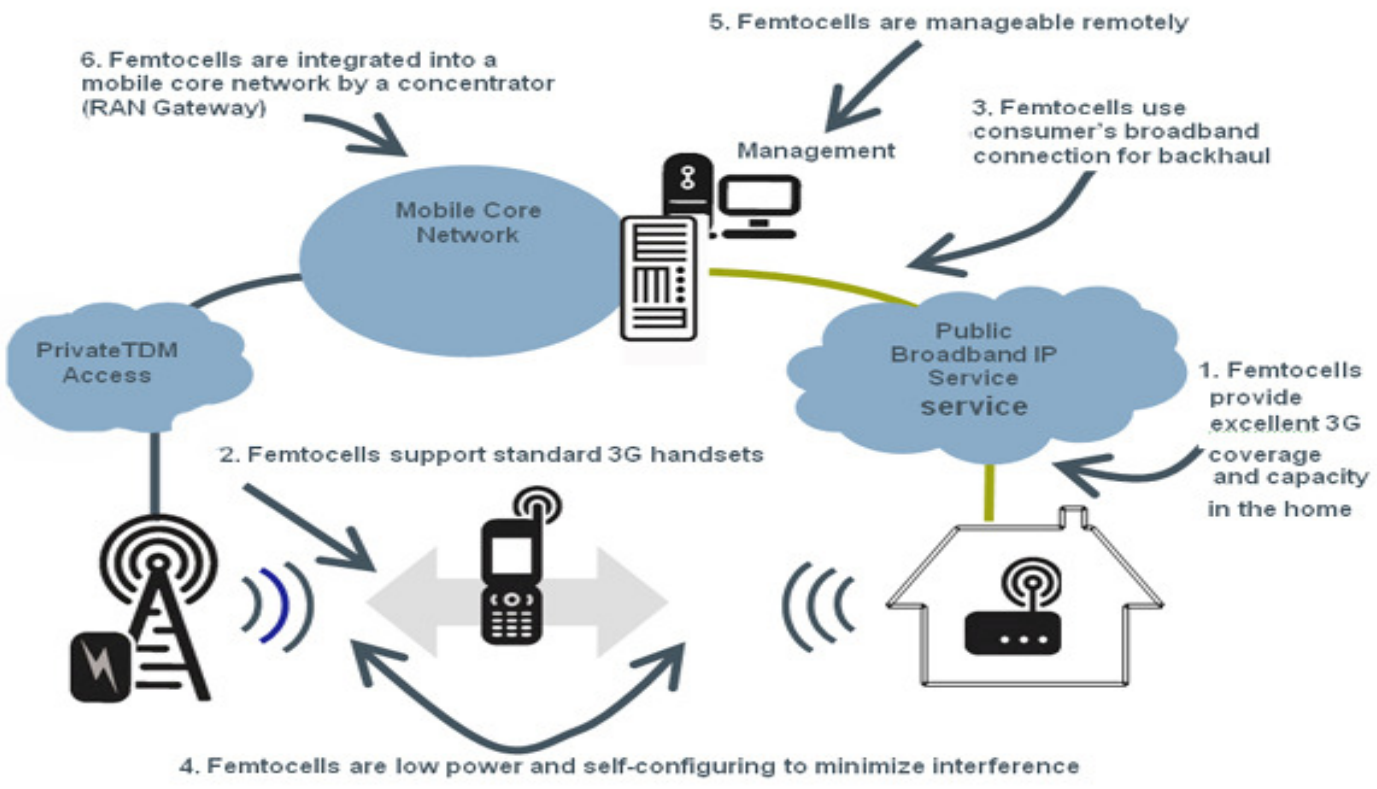

Femtocells working principle

Femtocells coupled with feature of self organizing systems, coverage and capacity optimization, software upgradations and handover optimization are supported in $802.16 \mathrm{~m}$ to maximize overall network parameters. The scope of self organizing networks in $802.16 \mathrm{~m}$ is limited to the measurement and reporting of air interface performance metrics, and the subsequent adjustments. Self Organization can be divided into two:

- Initializing and configuring Base Stations automatically with minimum human

- Self-optimization from the BS/MS and fine tuning the BS parameters in order to optimize the network performance which includes QoS, network efficiency, throughput, cell coverage and cell capacity.

\subsection{Backward Compatibility and coexistence:}

IEEE $802.16 \mathrm{~m}$ base stations will support interoperability with $802.16 \mathrm{e}$ systems. An IEEE $802.16 \mathrm{~m}$ mobile station will operate with $802.16 \mathrm{e}$ at performance similar 802.16 mobile station. An $802.16 \mathrm{~m}$ capable bas station will support interworking with $802.16 \mathrm{~m}$ and $802.16 \mathrm{e}$ devices.

\subsection{Multi Carrier Aggregation:}

Flexible spectrum use is achieved through use of scalable OFDMA multiple access scheme, (Scalable OFDMA is a version of OFDMA in which the sub carrier spacing is independent of bandwidth and the number of subcarriers scales with bandwidth) in the DL and UL tone dropping 
techniques in OFDMA, as well as use of one of multiple RF carriers. Multiple component carriers can be aggregated upto to $100 \mathrm{MHZ}$ transmission bandwidth. The aggregated component carriers can be either contiguous or non-contiguous in the frequency domain. Channel bandwidth of 5, 10, 20 and 40(optional) is supported in $16 \mathrm{~m}$ with multi carrier aggregation up to $100 \mathrm{MHZ}$.

\section{CONCLUSION}

This paper gives an overview of the PHY and MAC layer specifications of the IEEE $802.16 \mathrm{~m}$ standard also known as WiMAX Rel. 2.0. It gives various other details on the IEEE $802.16 \mathrm{~m}$ standard which are in accordance of the IMT-Advanced specifications, given by the ITU. With the IEEE $802.16 \mathrm{~m}$ update the WiMAX mobile technology is expected to be a candidate for $4 \mathrm{G}$ systems, as it meets the specifications under IMT-Advanced. For high bandwidth demand of the future wireless networks IEEE $802.16 \mathrm{~m}$ is a strong candidate, expected to provide data rates in the range of 1 Gbps for high speed mobile users.

\section{REFERENCE}

[1] Ramjee Prasad, Fernando J. Velez, "WiMAX Networks: Techno Economic Vision and Challenges, “, Springer Books, http://www.springerlink.com/content/978-90-481-8752-2\#section=714106\&page=1

[2] Etemad, K., "Overview of mobile WiMAX technology and evolution", Communications magazine, IEEE, vol. 46, p31-40, 2008

[3] A. Ghosh., "Broadband Wireless Access with WiMAX /802.16: Current Performance Benchmarks and Future Potential," IEEE Communication Magazine, vol. 43 p129-36, Feb. 2005,

[4] IEEE 802.16 -2009 "IEEE Standard for Local and Metropolitan Area Networks: Part 16: Air Interface for Broadband Wireless Access Systems, IEEE Std 802.16- 2009”, May 2009,

[5] Piggin P, "Emerging mobile WiMAX antenna technologies", Communications Engineer, vol. 4 p2933, 2006.

[6] Koon Hoo Teo, Zhifeng Tao, Jinyun Zhang,"The Mobile Broadband WiMAX standard[Standards in Nutshell]”, Signal Processing Magazine, IEEE, p144-148, 2007.

[7] C. So-In, R. Jain, and A. Al-Tamimi, "Scheduling in IEEE 802.16e WiMAX Networks: Key issues and a survey,"IEEE J. Select. Areas Communication., vol. 27, no. 2, p156-171, Feb. 2009.

[8] Q. Ni, "Investigation of bandwidth request mechanisms under point-to-multipoint mode of WiMAX networks," IEEE Communication Magazine, vol. 45, no. 5, p132-138, May 2007.

[9] Kim, W, "Mobile WiMAX, the leader of the mobile Internet era [WiMAX Report]", Communications Magazine, IEEE, vol. 47, Issue 6, p10-12, 2009.

[10] IEEE 802.16-2004, "Local and Metropolitan Networks — Part 16: Air Interface for Fixed Broadband Wireless Access Systems," 2004.

[11] IEEE 802.16e-2005, "Local and Metropolitan Networks - Part 16: Air Interface for Fixed and Mobile Broadband Wireless Access Systems, Amendment 2: Physical and Medium Access Control Layers for Combined Fixed and Mobile Operation in Licensed Bands and Corrigendum 1," 2006.

[12] Yang Yang, Honglin Hu and Jing Xu "Relay Technologies for WiMAX and LTE-Advanced Mobile Systems" IEEE Communications Magazine, p100-105, October 2009,

[13] BP Tiwari, "WiMAX 2.0 for operator", Whitepaper, www.wimax.com/whitepapers/bp-tiwari-wimax20-for-operators.pdf,

[14] Green Packet, "WiMAX, a Wireless Solution for Fixed Wireless Access in Emerging Markets", http://www.wimax.com/whitepapers/green-packet-wimax-wireless-solution-for-fixed-wirelessaccess.pdf

[15] Analysis of WiMAX Data Rate Performance, http://www.wimax.com/whitepapers/university-oflisbon-analysis-of-wimax-data-rate-performance.pdf

[16] Mobile WiMAX Update and IEEE $802.16 \mathrm{~m}$, http://ewh.ieee.org/r6/scv/comsoc/Talk_032509_WiMAXUpdate.pdf

[17] WiMAX Technologies: Architectures, Protocols, Resource, http://www.iaria.org/conferences2008/filesCTRQ08/CTRQ_2008_WiMAX_tutorial_EB-v1.3.pdf 